\title{
Commentaire
}

\section{La « santé publique et des populations » au Canada va-t-elle à l'avenir s'unir ou se diviser? Réflexions de l'intérieur}

\author{
Kelsey Lucyk, M. Sc.; Lindsay McLaren, Ph. D.
}

Cet article a fait l'objet d'une évaluation par les pairs.

Diffuser cet article sur Twitter

\section{Introduction}

« Est-ce que la santé publique et des populations constitue véritablement un domaine unifié, ou est-ce que la santé des populations s'est simplement agrégée à la santé publique pour gagner en crédibilité? »

Le commentaire présenté ici résulte de cette question, posée à Kelsey Lucyk (KL) dans le cadre de son examen d'admission au doctorat. En réponse, KL a cité les récents développements dans le domaine pour justifier de sa conviction que la santé publique et des populations (SPP) constitue bel et bien une discipline unifiée. Toutefois, au fil des conversations des semaines et des mois qui ont suivi, nous avons conclu que cet enjeu allait bien audelà de l'étiquetage de ministères et d'organismes de "santé publique et des populations » et qu'en débattre pouvait être pertinent, particulièrement pour la prochaine génération de chercheurs en SPP. Dans ce commentaire, nous faisons la démonstration 1) que parfois l'expression SPP sous-entend une cohérence d'idées, de valeurs et de principes qui n'est pas forcément réalisée, 2) qu'il est temps et important de viser à une SPP plus unifiée et 3) que l'unification de la SPP offre à la fois des défis et des opportunités, ce que nous illustrons dans les domaines du financement de la recherche, des effectifs en santé publique et de l'éthique en SPP.

\section{Argument $n^{0} 1$ : l'expression SPP sous-entend une cohérence qui n'est pas forcément réalisée}

D'après notre expérience, l'expression SPP donne parfois l'impression d'une cohérence d'idées, de valeurs et de principes qui n'est cependant pas toujours réalisée. Cette impression relève de divers éléments, par exemple de l'existence de programmes de formation diplômante dans les universités de Calgary ${ }^{1}$, de Vancouver $^{2}$, d'Ottawa ${ }^{3}$ et de Waterloo ${ }^{4}$, tout comme l'existence de ministères de SPP au sein des réseaux de la santé5,6 ou encore du fait de divers événements (voir tableau 1). Néanmoins, en pratique, cette cohérence n'est pas toujours réalisée. KL se souvient par exemple d'un étudiant postdoctoral rencontré à une réunion nationale sur la santé publique qui a fait observer que l'on était habitué à ignorer ce qu'est la "santé des populations » et à ne parler que de "santé publique ", ce qui implique que ces deux concepts sont identiques - du moins dans une certaine mesure ou pour des publics précis. L'expérience de KL va dans le sens inverse : en tant qu'universitaire, elle se décrit comme une " chercheuse en santé publique et des populations ", mais ses collègues du domaine de la santé publique ne la considèrent pas comme une "vraie personne du milieu de la santé publique » parce qu'elle n'a pas de diplôme en santé. Il demeure donc nécessaire de clarifier les limites et l'avenir de la SPP, particulièrement en raison du nombre croissant d'étudiants dans ce domaine.

\section{Argument $n^{0} 2$ : il est temps et important de viser à une SPP plus unifiée}

L'une des questions fondamentales formant le cœur de notre commentaire est de savoir si la SPP devrait être une discipline unifiée. Certains jugent que la réponse est «non $»^{7}$. D’importants points sont à relever parmi les arguments avancés contre l'unification de la SPP, en particulier

\section{Points saillants}

- En dépit de l'intégration prévue de la "santé publique et des populations » (SPP), divers problèmes en matière de financement de la recherche, d'effectifs en santé publique ainsi que d'éthique constituent des défis à l'unification du domaine.

- Les auteurs soutiennent que surmonter ces défis est un objectif fort louable pour le bien-être futur de la population canadienne.

l'inquiétude que la SPP ne soit pas efficace si sa portée est trop large, ou que son unification dilue l'urgence de la santé publique?

Nous ne sommes pas d'accord avec cette position, et nous jugeons qu'il est temps et important de viser à une SPP plus unifiée. C'est important, car intégrer les déterminants sociaux de la santé et penser de façon critique aux inégalités en matière de santé - ce que la SPP vise à faire ${ }^{8}$ - est nécessaire dans le cadre d'une conception holistique de la santé, ainsi que pour éliminer les cloisonnements professionnels et organisationnels qui empêchent toute intervention intersectorielle en santé ou en équité en santé. Dans certains cas, l'élimination de ces cloisonnements va exiger des changements importants dans le réseau en santé publique. Par exemple, la " santé » possède actuellement son propre ministère au sein de plusieurs provinces canadiennes (comme Alberta Health) et à l'échelle fédérale (Santé Canada), ce qui implique une séparation vis-à-vis d'autres 
déterminants du bien-être, alors qu'auparavant on usait d'une portée plus générale (que ce soit le ministère fédéral des Pensions et de la Santé nationale [1928] ou le ministère fédéral de la Santé nationale et du Bien-être social [1944]) ${ }^{9,10}$.

Il est temps de viser à une SPP plus unifiée. Contrairement à il y a 20 ans, les universités canadiennes offrent maintenant de nombreux programmes aux étudiants qui n'ont pas nécessairement l'intention de se diriger vers le domaine de la santé publique au sens classique (p. ex. services infirmiers de santé publique, santé publique et médecine préventive) mais souhaitent plutôt poursuivre une carrière universitaire ou appliquer les principes de la SPP dans d'autres secteurs. Le programme de baccalauréat en sciences de la santé offert par l'Université de Calgary - et plus précisément sa spécialisation en santé et société en constitue un excellent exemple. Cette tendance relativement récente nous convient tout à fait : si nous sommes tous deux attirés par l'idée d'une SPP unifiée, c'est parce qu'elle réunit la santé, les sciences sociales et les sciences humaines, comme ce fut largement le cas, fut un temps, dans le secteur de la santé et dans les professions de la santé.

\section{Argument n ${ }^{0} 3$ : l'unification de la SPP offre des défis comme des opportunités}

Pour alimenter la réflexion sur la SPP, nous avons sélectionné trois thèmes (parmi de nombreux autres) qui semblent créer un clivage dans ce milieu : le financement de la recherche, les effectifs en santé publique et l'éthique en SPP. Nous avons déterminé pour chacun, dans l'intention de lancer le dialogue, ce qui, à notre avis, en constitue les principaux défis et opportunités.

\section{Financement de la recherche}

Défi : L'annonce faite en 2009 par le Conseil de recherches en sciences humaines qu'il ne financerait plus la recherche en santé a constitué un défi pour la SPP en tant que domaine interdisciplinaire, puisque bon nombre de ses spécialistes en sciences sociales ont dû se démener dans le paysage et les diverses procédures de financement des Instituts de recherche en santé du Canada (IRSC) ${ }^{11}$. Ce changement a mis en lumière les différences entre les normes et les attentes liées aux sciences sociales et celles liées à la recherche traditionnelle en santé (en particulier la structure des demandes de subvention de recherche, la paternité d'une œuvre, la durée et le rythme des publications et l'accent sur la théorie) ${ }^{12}$ Elle a également révélé quels domaines de recherche étaient considérés comme viables et pertinents. Ces différences désavantageront surtout ceux qui sont les plus à même de fournir à la SPP un savoir théorique et critique riche.

Opportunité : Il est essentiel d'intégrer les sciences sociales et de la santé à la SPP. En tant qu'organisme national de financement et instance de direction de la recherche en santé au Canada, les IRSC offrent un lieu où aborder les défis de cette intégration. L'Institut de la santé publique et des populations (ISPP) en constitue un bon exemple, avec les efforts importants qu'il a déployés pour modifier le paysage des évaluations par les pairs afin que les candidats interdisciplinaires soient évalués de façon juste et transparente par des examinateurs possédant l'expertise nécessaire, grâce à des concours précis et axés sur les priorités ${ }^{13}$. Bien que les défis mentionnés plus haut n’aient pas été éliminés, il semble que d'importants progrès aient été réalisés.

\section{Effectifs en santé publique}

Défi : Les effectifs en santé publique (médecins, inspecteurs en santé publique, personnel de laboratoires, personnel infirmier) demeurent principalement employés dans le secteur de la santé (organismes de services de santé ou ministères de la Santé). Cette structure complique la prise de mesures liées aux déterminants sociaux de la santé et de l'équité ainsi qu'à l'équité en santé, les deux grands thèmes fondateurs de la SPP, ce qui fait qu'elle sort du champ d'application des cadres législatif et réglementaire de la santé publique. La prise de mesures liées aux déterminants sociaux de la santé est susceptible de sortir du cadre de travail courant en santé publique consistuant à offrir des programmes et des services au public ${ }^{14}$. De plus, le cadre législatif qui définit la santé publique dans les diverses provinces pourrait ne pas être compatible avec une SPP intégrative. Par exemple, le document Public Health Act : Revised Statues of Alberta $2000^{15}$ de la province de l'Alberta ne fait aucune mention des déterminants sociaux de la santé, ni même des maladies chroniques. Ces questions pourraient créer un clivage entre les nombreux experts assumant des fonctions clés en santé publique (p. ex. en prévention des maladies, plus particulièrement des maladies transmissibles) et l'objectif affirmé de la SPP de contribuer de manière élargie à la santé des populations (p. ex. au moyen d'interventions politiques et sociales, en dehors du réseau de la santé).

Opportunité : Malgré ces sources de clivage, de grandes possibilités s'offrent et, dans certains cas, des progrès ont été faits au sein des organismes professionnels et de réglementation en santé publique afin d'unifier davantage le domaine. Par exemple, Brassolotto, Raphael et Baldeo ${ }^{14}$ ont documenté le fait que certains services en santé de l'Ontario, en plus d'offrir des services traditionnels en santé publique, font activement la promotion des déterminants sociaux de la santé et prennent des mesures concrètes à cet égard. Ainsi, Santé publique Ontario a entrepris de s'atteler aux déterminants de la santé et de réduire les inégalités en matière de santé avec les Normes de santé publique de l'Ontario ${ }^{16}$.

Des progrès législatifs peuvent également être constatés dans certaines provinces. En Colombie-Britannique, le Public Health Act (SBC 2008) inclut les maladies chroniques dans la liste des obstacles à la santé, ce qui - du moins en théorie - permet au ministre de tenir compte des déterminants sociaux de la santé ou de considérations en matière d'équité en santé au moment d'élaborer un plan visant à en définir, prévenir et atténuer les conséquences négatives ${ }^{17}$. La Loi sur la santé publique (S-2.2) du Québec va plus loin, en permettant au ministre de la Santé, au directeur de la Santé publique et aux institutions d'intervenir dans la prévention des maladies et des traumatismes ainsi que de prendre en compte les " problèmes sociaux ayant un impact sur la santé de la population $»^{18}$ en agissant sur les déterminants sociaux de la santé. Ainsi, Le Québec promeut et met en œuvre des politiques publiques saines en utilisant des études d'impact sur la santé19. Enfin, dans les dernières années, l'Agence de la santé publique du Canada a tenté de cerner les effectifs sans cesse plus nombreux de la SSP au moyen de compétences essentielles en santé publique ainsi qu'en harmonisant les données sur les différentes formations postsecondaires et de cycles supérieurs offertes dans le domaine de la SPP ${ }^{20,21}$. Cela permet de mieux comprendre quelles spécificités de la SPP permettent des interventions intersectorielles et les renforcent, 
TABLEAU 1

Chronologie des principaux événements liés à l'évolution de la «santé publique et des populations », 1974 à 2004

\begin{tabular}{|c|c|c|}
\hline Année & Événement & Contribution au domaine de la SPP \\
\hline 1974 & Publication du Rapport Lalonde ${ }^{32}$ & Influe sur le nombre de développements en promotion de la santé. \\
\hline 1975 & $\begin{array}{l}\text { Création du Programme national de recherche et de développement en } \\
\text { matière de santé }\end{array}$ & Stimule et appuie la recherche sur les questions de santé nationale. \\
\hline $\begin{array}{c}1978 \\
\text { (R.-U.) }\end{array}$ & $\begin{array}{l}\text { Publication des conclusions de Marmot, Rose, Shipley et Hamilton }{ }^{33} \text { à la } \\
\text { suite de Whitehall I }\end{array}$ & $\begin{array}{l}\text { Introduit la notion de gradient social dans le domaine de la recherche } \\
\text { épidémiologique. }\end{array}$ \\
\hline $\begin{array}{c}1982 \\
(\mathrm{CAN})\end{array}$ & Création de l’Institut canadien des recherches avancées & $\begin{array}{l}\text { Sert « d'organe de réflexion » pour élaborer de nouveaux cadres } \\
\text { conceptuels. }\end{array}$ \\
\hline $\begin{array}{l}1985 \\
\text { (R.-U.) }\end{array}$ & Publication de Sick Individuals and Sick Populations par Rose ${ }^{34}$ & Présente la stratégie populationnelle de prévention. \\
\hline $\begin{array}{c}1986 \\
\text { (Intl.) }\end{array}$ & Publication de la Charte d’Ottawa pour la promotion de la santéé & $\begin{array}{l}\text { Facilite les développements en matière de promotion de la santé et } \\
\text { présente les prérequis pour la santé. }\end{array}$ \\
\hline$(\mathrm{CAN})$ & Publication du Rapport Epp ${ }^{36}$ & $\begin{array}{l}\text { Les ministères canadiens commencent à adopter la promotion de la santé } \\
\text { dans leurs programmes. }\end{array}$ \\
\hline $\begin{array}{l}1987 \\
(\mathrm{CAN})\end{array}$ & $\begin{array}{l}\text { Création d'un programme sur la santé des populations par l'Institut } \\
\text { canadien des recherches avancées }\end{array}$ & $\begin{array}{l}\text { Tient compte des changements apportés au gouvernement et dans la SPP; } \\
\text { la santé publique passe graduellement de la promotion de la santé à la } \\
\text { santé des populations. }\end{array}$ \\
\hline $\begin{array}{r}1989 \\
(\mathrm{CAN})\end{array}$ & $\begin{array}{l}\text { Présentation d'un concept de la santé des populations par l'Institut } \\
\text { canadien des recherches avancées }\end{array}$ & Tient compte de l'interaction complexe des déterminants de la santé. \\
\hline $\begin{array}{l}1991 \\
(\mathrm{CAN})\end{array}$ & Publication de The Determinants of Health par Mustard et Frank ${ }^{37}$ & $\begin{array}{l}\text { Arrive à la conclusion que les grands déterminants de la santé sont hors } \\
\text { de la portée du système des soins de santé, tant à l'échelle des individus } \\
\text { que de la population. }\end{array}$ \\
\hline $\begin{array}{c}1991 \\
\text { (R.-U.) }\end{array}$ & $\begin{array}{l}\text { Publication des conclusions de Marmot, Davey Smith, Stansfeld et coll. }{ }^{38} \\
\text { à la suite de Whitehall II }\end{array}$ & $\begin{array}{l}\text { Met l'inégalité en matière de santé à l'avant-plan de la recherche à } \\
\text { l'échelle de la population. }\end{array}$ \\
\hline $\begin{array}{c}1994 \\
(\mathrm{CAN})\end{array}$ & $\begin{array}{l}\text { Publication de Why are Some People Healthy and Others Not? par Evans, } \\
\text { Barer et Marmor }{ }^{39}\end{array}$ & $\begin{array}{l}\text { Offre du soutien épidémiologique afin d'expliquer l'influence des facteurs } \\
\text { sociaux et économiques sur la santé. }\end{array}$ \\
\hline $\begin{array}{l}1994 \\
(\mathrm{CAN})\end{array}$ & $\begin{array}{l}\text { Publication de Stratégies d’amélioration de la santé de la population : } \\
\text { investir dans la santé des canadiens par les ministre fédéral, provinciaux et } \\
\text { territoriaux de la Santé }{ }^{40}\end{array}$ & $\begin{array}{l}\text { L'approche axée sur la santé de la population est officiellement adoptée } \\
\text { par les gouvernements. }\end{array}$ \\
\hline $\begin{array}{l}1996 \\
(\mathrm{CAN})\end{array}$ & 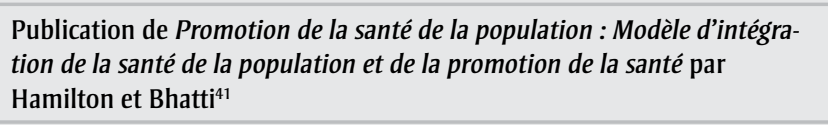 & $\begin{array}{l}\text { Réunit les concepts de la santé de la population et de la promotion } \\
\text { de la santé. }\end{array}$ \\
\hline $\begin{array}{c}1997 \\
(\mathrm{CAN})\end{array}$ & $\begin{array}{l}\text { Mise sur pied du Comité consultatif fédéral-provincial-territorial sur la } \\
\text { santé de la population }\end{array}$ & Définit la santé des populations à l'échelle gouvernementale. \\
\hline $\begin{array}{c}1998 \\
(\mathrm{CAN})\end{array}$ & $\begin{array}{l}\text { Publication de Population health in Canada : a systematic review par } \\
\text { Hayes et Dunn }{ }^{42}\end{array}$ & $\begin{array}{l}\text { Détermine différentes façons possibles de concevoir la santé des } \\
\text { populations, en tant que point de vue, recherche, cadre ou approche. }\end{array}$ \\
\hline $\begin{array}{c}1998 \\
(\mathrm{CAN})\end{array}$ & $\begin{array}{l}\text { Publication de Wealth, Equity and Health Care: A Critique of a "Popula- } \\
\text { tion Health" Perspective on the Determinants of Health par Poland, } \\
\text { Coburn, Robertson et Eakin }{ }^{43}\end{array}$ & $\begin{array}{l}\text { Reproche au modèle de santé des populations d'être athéorique et } \\
\text { réductionniste. }\end{array}$ \\
\hline $\begin{array}{c}2000 \\
(\dot{E} .-U .)\end{array}$ & $\begin{array}{l}\text { Le National Committee on Vital Health and Statistics des Centers for } \\
\text { Disease Control and Prevention des États-Unis envisage le concept de la } \\
\text { santé des populations mis de l'avant par l'Institut canadien des recherches } \\
\text { avancées dans sa vision pour les statistiques en matière de santé }\end{array}$ & $\begin{array}{l}\text { Montre que le concept de santé des populations s'étend à l'échelle } \\
\text { internationale. }\end{array}$ \\
\hline $\begin{array}{c}2000 \\
(\mathrm{CAN})\end{array}$ & $\begin{array}{l}\text { Création des Instituts de recherche en santé du Canada par une loi } \\
\text { fédérale, en remplacement du Programme national de recherche et de } \\
\text { développement en matière de santé }\end{array}$ & Inclut l'Institut de recherche sur la santé des populations en 2000. \\
\hline $\begin{array}{c}2001 \\
(\mathrm{CAN})\end{array}$ & $\begin{array}{l}\text { Article faisant état de la prise de position par la Direction générale de la } \\
\text { promotion des programmes de la santé de Santé Canada, à l'intention du } \\
\text { personnel en promotion de la santé }\end{array}$ & $\begin{array}{l}\text { L'approche axée sur la santé de la population est adoptée par Santé } \\
\text { Canada en tant que force unificatrice pour l'ensemble des interventions } \\
\text { du système de santé. }\end{array}$ \\
\hline $\begin{array}{l}2003 \\
(\mathrm{CAN})\end{array}$ & Publication de Population Health in Canada : A Brief Critique par Coburn ${ }^{44}$ & $\begin{array}{l}\text { Reconnaît que la promotion de la santé a été « détrônée » par la santé des } \\
\text { populations en tant que discours politique crédible sur la santé. }\end{array}$ \\
\hline $\begin{array}{l}2004 \\
(\mathrm{CAN})\end{array}$ & Création de l’Agence de la santé publique du Canada & $\begin{array}{l}\text { Adopte une approche axée sur la santé des populations et crée des } \\
\text { bureaux régionaux pour que la Direction générale de santé de la } \\
\text { population et de la santé publique la diffuse. }\end{array}$ \\
\hline
\end{tabular}

Abréviations : CAN, Canada; Intl, International; R.-U., Royaume-Uni; SPP, santé publique et des populations. 
grâce à des effectifs et à un champ d'activité en SPP plus harmonieux.

\section{Tentatives de consolidation des assises éthiques de la SPP}

Défi : Comme la pratique de la santé publique est principalement présente au sein du système de soins de santé, ses lignes directrices en matière d'éthique ont toujours été définies par des principes bioéthiques (p. ex. l'autonomie, la bienfaisance, la non-malfaisance, le respect des droits de la personne) et orientées par la théorie morale de l'utilitarisme (p. ex. le bien public $)^{22}$. Cependant, comme cela a déjà été signalée ${ }^{23,24}$, ces principes bioéthiques se sont avérés inadéquats pour surmonter complètement les défis de la SPP en matière d'interventions structurelles visant des populations entières, ces interventions pouvant entrer en conflit avec la volonté du public à propos des bienfaits pour la population (p. ex. la fluoration de l'eau collective). Cette tension a conduit à la création de sous-disciplines essentielles (comme l'éthique en santé publique) pour promouvoir l'avancement de la pensée éthique de façon à répondre à ce besoin (p. ex. le modèle de gérance du Nuffield Council on Bioethics). ${ }^{25}$

Opportunité : On constate une tendance prometteuse à acquérir les connaissances essentielles sur certains des défis spécifiques liés aux interventions en santé des populations dans un cadre d'éthique en santé publique. Par exemple, les spécialistes débattent sur les mérites et les inconvénients de mener des interventions en SPP à l'échelle de la population, ou généralisées : d'un côté, on évoque les éventuelles conséquences négatives d'une approche à cette échelle ${ }^{26,27}$, de l'autre, on montre les profits et la contribution potentielle à l'équité de cette approche ${ }^{28}$. L'assise intellectuelle de la SPP va être de plus en plus robuste grâce à ces travaux. De même, on a vu naître des cadres éthiques dans lesquels les caractéristiques de la santé des populations sont mieux incorporées, répondant au besoin du milieu en matière de transparence et de régulation minimale, d'équité et de justice sociale..$^{23,29,31}$ Ces travaux peuvent contribuer à une meilleure unification de la SPP, car on commence à s'attaquer à la question de l'équilibre entre l'aspect utilitaire de la santé publique - que beaucoup considèrent comme son principal atout - et les possibles conséquences imprévues - dont il faut tenir compte - de cette approche destinée à améliorer la santé de tous.

\section{Conclusion}

$\mathrm{Au}$ fur et à mesure que la SPP va évoluer au cours du $21^{\text {e }}$ siècle et que les inscriptions aux programmes interdisciplinaires de cycle supérieur en "santé publique et des populations » vont se multiplier, nous sommes persuadés que la question de savoir s'il faut intégrer la SPP et comment l'unifier restera tout aussi pertinente et importante. Nous sommes conscients du fait que les thèmes que nous avons traités dans ce texte (la recherche, les effectifs en santé publique et l'éthique en SPP) ne sont pas exclusifs les uns des autres et ne constituent que des exemples parmi d'autres.

Nous encourageons les études et les discussions sur ce sujet, et nous espérons que cet article stimulera les débats et discussions parmi les dirigeants, les travailleurs et les étudiants en SPP.

\section{Remerciements}

Nous désirons remercier Dre Margaret Russell, qui a posé la question ayant donné lieu à cette réflexion. Le travail de Kelsey Lucyk est subventionné par une bourse d'études pour gradués d'Alberta Innovates-Health Solutions. Le travail de Lindsay McLaren est subventionné par une Chaire en santé publique appliquée (voir http:// www.cihr-irsc.gc.ca/f/49128.html), financée par les Instituts de recherche en santé du Canada (Institut de la santé publique et des populations; Institut de l'appareil locomoteur et de l'arthrite), l'Agence de la santé publique du Canada et Alberta Innovates-Health Solutions.

\section{Références}

1. Université de Calgary. CHS Graduate Student Competencies and Requirements in Population and Public Health. Calgary (AB) : Université de Calgary; 2017. En ligne à : http://wcm .ucalgary.ca/gse/files/gse/competencies _population_public_health_2017.pdf

2. Université de la Colombie-Britannique, School of Population and Public Health, Maîtrise en hygiène publique (MHP) [Internet]. Vancouver : Université de la Colombie-Britannique; sans date [consulté le 4 avril 2017]. En ligne à https://www.grad.ubc.ca /prospective-students/graduate-degree -programs/master-of-public-health
3. Université d'Ottawa. Graduate and postdoctoral studies [Internet]. Ottawa (Ont.) : Université d'Ottawa sans date [consulté le 4 avril 2017]. En ligne à : http://www.grad.uottawa.ca/Default .aspx?tabid $=1727 \&$ page $=$ SubjectDetails \&Kind $=$ H\&SubjectId $=97$

4. Université de Waterloo. School of Public Health and Health Systems. Waterloo (Ont) : Université de Waterloo; sans date [consulté le 4 avril 2017]. En ligne à : https://uwaterloo.ca/public -health-and-health-systems/future -graduate-students/research-based -programs/public-health-and-health -systems-phd

5. Alberta Health Services. Population, public and indigenous health: strategic clinical network. Edmonton (AB) : Alberta Health Services; 2017 [consulté le 4 avril 2017]. En ligne à : http://www.albertahealthservices.ca /scns/Page13061.aspx

6. Regina Qu'Appelle Health Region. Population and Public Health. Regina (SK) : Regina Qu'Appelle Health Region; 2015 [consulté le 4 avril 2017]. En ligne à : http://www.rqhealth.ca /departments/population-and-public -health

7. Rothstein M. Rethinking the meaning of public health. J Law Med Ethics. 2002;30(2):144-149.

8. Frank J. Why "population health"? Can J Public Health. 1995;86(3): 162-164.

9. Cheung-Gertler J. Santé Canada. Dans L'encyclopédie canadienne. Toronto (Ont.) : Historica Canada; 2008 [mise à jour le 5 août 2014; consulté le 4 avril 2017]. En ligne à : http:// encyclopediecanadienne.ca/fr/article /sante-canada/

10. Agence de la santé publique du Canada. Histoire [Internet]. Ottawa (Ont.) : Gouvernement du Canada; 2008 [modifié le 4 avril 2008; consulté le 4 avril 2017]. En ligne à : http:// www.phac-aspc.gc.ca/about_apropos /history-fra.php 
11. Halbersma J. Les chercheurs en sciences sociales doivent dorénavant demander des subventions aux IRSC [Internet]. Affaires universitaires; 8 octobre 2014 [consulté le 4 avril 2017]. En ligne à : http://www.affairesuniversitaires.ca /opinion/a-mon-avis/les-chercheurs-en -sciences-sociales-doivent-dorenavant -demander-subventions-aux-irsc/\#_ga $=1.14002359 .1884578313 .1491960308$

12. Albert K. Erasing the social from social science: the intellectual costs of boundary-work and the Canadian Institute of Health Research. Canadian Journal of Sociology. 2014;39(3):393-420.

13. Instituts de recherche en santé du Canada (IRSC). " Modifications aux instituts et réforme des programmes ouverts et de l'évaluation par les pairs » [Internet]. Ottawa (Ont.) IRSC; 2014 [mise à jour le 22 décembre 2014; consulté le 4 avril 2017]. En ligne à : http://www.cihr-irsc.gc.ca/f/48930 .html

14. Brassolotto J, Raphael D, Baldeo N. Epistemological barriers to addressing the social determinants of health among public health professionals in Ontario, Canada: a qualitative inquiry, Critical Public Health 2014;24(3): 321-336.

15. Province de l'Alberta. "Public Health Act, Revised Statutes of Alberta 2000 ", chapitre P-37. Edmonton (AB) : Imprimeur de la Reine pour l'Alberta; 2016.

16. Ministère de la Santé et des Soins de longue durée de l'Ontario. Normes de santé publique de l'Ontario, 2008 [révision en mai 2016]. Toronto (Ont.) : Imprimeur de la Reine pour l'Ontario; 2016. [publication no 020646]

17. Province de la Colombie-Britannique. Public Health Act, SBC 2008, chapitre 28. Victoria (C.-B.) : Imprimeur de la Reine; 2008 [mise à jour le 8 mars 2017; consulté le 4 avril 2017]. En ligne à : http://www.bclaws.ca/civix /document/id/complete/statreg/00 _08028_01

18. Gouvernement du Québec. Loi sur la santé publique, chapitre S-2.2, Québec (QC) : Éditeur officiel du Québec; 2016 [mise à jour].
19. Institut national de santé publique du Québec. Évaluation d'impact sur la santé. Montréal (QC) : Institut national de santé publique Québec; sans date [consulté le 4 avril 2017]. En ligne à : http://politiquespubliques.inspq.qc.ca /fr/evalutaion.html

20. Agence de la santé publique du Canada (ASPC). Compétences essentielles en santé publique au Canada : version 1.0. Ottawa (Ont) : ASPC; 2008. [no HP551/2008]

21. Agence de la santé publique du Canada (ASPC). Planifiez votre carrière en santé publique [Internet]. Ottawa (Ont.) : ASPC; sans date [modifié le 25 juillet 2016; consulté le 4 avril 2017]. En ligne à : http://www.phac-aspc.gc.ca/php -psp/training-fra.php

22. Kass N. An ethics framework for public health. American Journal of Public Health. 2001;91(11):1776-1782.

23. Upshur RE. Principles for the justification of public health intervention. Canadian Journal of Public Health. 2002; 93(2):101-103.

24. Scott L, Hatfield J, McIntyre L. A scoping review of unintended harm associated with public health interventions: towards a typology and an understanding of underlying factors. Canadian Journal of Public Health. 2014;59(1):3-14.

25. Nuffield Council on Bioethics. Public health: ethical issues. Londres (R.U.) : Cambridge Publishers Ltd.; 2007. En ligne à : http://nuffieldbioethics .org/wp-content/uploads/2014/07 /Public-health-ethical-issues.pdf

26. Ramos Salas X. The ineffectiveness and unintended consequences of the public health war on obesity. Canadian Journal of Public Health. 2015;106(2): e79-e81.

27. Bell K, McCullough L, Salmon A, Bell J. "Every space is claimed": smokers experiences of tobacco denormalisation. Sociology of Health and Illness. 2010;32(6):914-929.

28. McLaren L, McIntyre L, Kirkpatrick S. Rose's population strategy of prevention need not increase social inequalities in health. International Journal of Epidemiology. 2010;39(2):372-377.
29. Daniels N. Equity and population health: toward a broader bioethics agenda. Hastings Center Report, 2006; 36(4):22-35.

30. Braveman P, Gruskin S. Defining equity in health. Journal of Epidemiology and Community Health. 2003;57(4): 254-258.

31. Faden RR, Powers M. Health inequities and social justice: the moral foundations of public health. Bundesgesundheitsblatt Gesundheitsforschung Gesundheitsschutz. 2008;51(2):151-157.

32. Lalonde, M. Nouvelle perspective de la santé des Canadiens : Document de travail. Ottawa (Ont.) : Santé et Bien-être social Canada, 1974. [no catalogue : H31-1374]

33. Marmot M, Rose G, Shipley M, Hamilton P. Employment grade and coronary heart disease in British civil servants. Journal of Epidemiology and Community Health. 1978;32(4): 244-249.

34. Rose G. « Sick individuals and sick populations ». International Journal of Epidemiology. 1985;14(1):32-38.

35. Organisation mondiale de la Santé. Charte d'Ottawa pour la promotion de la santé. Genève $(\mathrm{CH})$ : Organisation mondiale de la Santé, 1986.

36. Epp J. Achieving health for all: A framework for health promotion. Ottawa (Ont) : Santé et Bien-être social Canada, 1986.

37. Mustard J, Frank J. The determinants of health. Toronto (Ont.) : Institut canadien des recherches avancées, programme sur la santé des populations; 1991.

38. Marmot M, Davey Smith G, Stansfeld $\mathrm{S}$, et al. Health inequalities among British civil servants: the Whitehall II study. Lancet. 1991; 337(8754):13871393

39. Evans R, Barer M, Marmor T (dir.). Why are some people healthy and others not? The determinants of health of populations. New York (NY) : Aldine de Gruyter; 1994. 378 p. 
40. Comité consultatif fédéral-provincialterritorial sur la santé de la population. Stratégies d'amélioration de la santé de la population : investir dans la santé des Canadiens. Ottawa (Ont.) : Santé Canada; 1994. [no H39-316/ 1994E]

41. Hamilton N, Bhatti T. Promotion de la santé de la population : Modèle d’intégration de la santé de la population et de la promotion de la santé [Internet]. Ottawa (Ont.) : Division du développement de la promotion de la santé; 1996 [consulté le 4 avril 2017]. En ligne à : http://www.phac -aspc.gc.ca/ph-sp/php-psp/index-fra .php

42. Hayes M, Dunn J. Population health in Canada: a systematic review. Ottawa (Ont.) : Canadian Policy Research Networks Inc.; 1998. [CPRN Study No. $\mathrm{H}|01|]$

43. Poland B, Coburn D, Robertson A, Eakin J. Wealth, equity and health care: a critique of a "population health" perspective on the determinants of health. Social Science and Medicine, 1998;46(7):785-798.

44. Coburn D, Denny K, Mykhalovskiy E, McDonough P, Robertson A, Love R. Population health in Canada : a brief critique. American Journal of Public Health. 2003;93(3):392-396. 